\title{
The second solar spectrum and the hidden magnetism
}

\author{
Jan O. Stenflo \\ Institute of Astronomy, ETH Zurich, HIT J 23.6, CH-8093 Zurich \\ email: stenflo@astro.phys.ethz.ch
}

\begin{abstract}
Applications of the Hanle effect have revealed the existence of vast amounts of "hidden" magnetic flux in the solar photosphere, which remains invisible to the Zeeman effect due to cancellations inside each spatial resolution element of the opposite-polarity contributions from this small-scale, tangled field. The Hanle effect is a coherency phenomenon that represents the magnetic modification of the linearly polarized spectrum of the Sun that is formed by coherent scattering processes. This so-called "Second Solar Spectrum" is as richly structured as the ordinary intensity spectrum, but the spectral structures look completely different and have different physical origins. One of the new diagnostic uses of this novel spectrum is to explore the magnetic field in previously inaccessible parameter domains. The earlier view that most of the magnetic flux in the photosphere is in the form of intermittent $\mathrm{kG}$ flux tubes with tiny filling factors has thereby been shattered. The whole photospheric volume instead appears to be seething with intermediately strong fields, of order $100 \mathrm{G}$, of significance for the overall energy balance of the solar atmosphere. According to the new paradigm the field behaves like a fractal with a high degree of self-similarity between the different scales. The magnetic structuring is expected to continue down to the $10 \mathrm{~m}$ scale, 4 orders of magnitude below the current spatial resolution limit.
\end{abstract}

Keywords. Sun: magnetic fields - Sun: photosphere - scattering - turbulence - atomic processes - line: formation - techniques: polarimetric

\section{Why magnetic flux on the Sun is hidden}

The impressive advances in angular resolution, from space with Hinode, from ground with the help of adaptive optics, allow us to explore ever smaller structures on the Sun. This brings fundamental insights into the basic astrophysical processes that govern the structuring, dynamics, and heating of stellar atmospheres. As however the structuring continues on scales that are far smaller than can be resolved in any foreseeable future even in solar observations, there is always a need for methods that allow us to extract information about the physics on scales that are too small to be resolved. This need has of course always been familiar to stellar physics, since stellar disks are normally unresolved (except for Doppler-Zeeman imaging of rapid rotators).

\subsection{The standard model}

Due to their finite angular resolution, all solar telescopes smear the true solar image with a smoothing window, the spatial resolution element. Solar magnetograms represent smoothed maps of the circular polarization produced by the longitudinal Zeeman effect. The early magnetograph recordings back in the 1960s showed that the apparent field strengths increased with the angular resolution of the instrument, so the question arose what the strength would be if we would have infinite resolution. With the introduction of the line-ratio technique in the early 1970s (Stenflo 1973) this question could be answered and it became clear that most (more than $90 \%$ ) of the magnetic flux on the quiet Sun 
seen in the magnetograms of that time (with a resolution of a few arcsec) came from intrinsically strong, 1-2 kG fields. Since the apparent field strengths in these line-ratio observations were of order $1-10 \mathrm{G}$, this finding implied that the $\mathrm{kG}$ flux elements were far smaller than the available resolution, so the interpretation had to be based on a parameterized model for the magnetic field. The simple 2-component model that was introduced was found to give consistent results not only for the line-ratio data (with various line combinations), but also for the rich sets of constraints provided by the FTS (Fourier Transform Spectrometer) circular-polarization spectra (Stenflo et al. 1984) and for the resolved Zeeman splittings in the near infrared, (cf. Rüedi et al. 1992) (with occasional extensions of the 2-component to a 3-component model).

The 2-component model assumed that we have one magnetic component with a certain filling factor, while the other component was assumed to be non-magnetic. The magnetic, $\mathrm{kG}$ component soon found its theoretical counterpart in MHD models of magnetic flux tubes (e.g. Spruit 1976). Including MHD constraints like self-consistent field expansion with height in the 2-component model, it became possible to construct semi-empirical flux-tube models with increasing levels of sophistication (cf. Solanki 1993).

It was however obvious already when the 2-component model was introduced nearly four decades ago that the concept of a "non-magnetic" component is non-physical and only introduced for the sake of mathematical and interpretational convenience. Due to the enormously high electrical conductivity of the turbulent photosphere it is physically inconceivable that anything can be "non-magnetic". What the term "non-magnetic" means in the diagnostic context is that this component does not contribute significantly to the net longitudinal Zeeman-effect polarization integrated over the resolution element.

The next challenge therefore became to find out what the intrinsic magnetic properties of the "non-magnetic" component are. Since the longitudinal Zeeman effect is "blind" to this component, another spectral signature had to be found, which could provide a glimpse of its elusive properties. Magnetic line broadening had insufficient sensitivity and could only set an upper limit to the field strength (Stenflo \& Lindegren 1977). The breakthrough came with the application of the Hanle effect (cf. Sect. 2.1) to an interpretational model based on a random, turbulent field. This led to the discovery of the existence of "hidden" (with respect to the Zeeman effect), turbulent fields with strengths in the range 10-100 G (Stenflo 1982).

The combination of line-ratio and Hanle data led to a "standard model" or "paradigm" for solar magnetism, as expressed in Fig. 1. The flux tube component expands rapidly with height, to satisfy the requirement of pressure balance, until the field fills the coronal volume. This component provides the flux that is visible in magnetograms (the circularpolarization maps of the longitudinal Zeeman effect). The component between the flux tubes contains a weaker, chaotically tangled "turbulent" field. Since the opposite magnetic polarities of this tangled field are mixed on subresolution scales, the positive and negative contributions to the circular polarization cancel out, so that this field becomes "hidden", i.e., invisible to the Zeeman effect.

\section{Second Solar Spectrum and the Hanle effect}

In contrast to the Zeeman effect, the Hanle effect is a coherence phenomenon that only occurs in coherent scattering processes. Such scattering produces polarization also in the absence of any magnetic fields, a familiar example being the polarization of the blue sky by Rayleigh scattering on molecules. The Sun's spectrum is polarized by coherent scattering, but the degree of polarization is small due to the small degree of anisotropy of the incident radiation field for scattering processes inside the Sun's atmosphere. It 


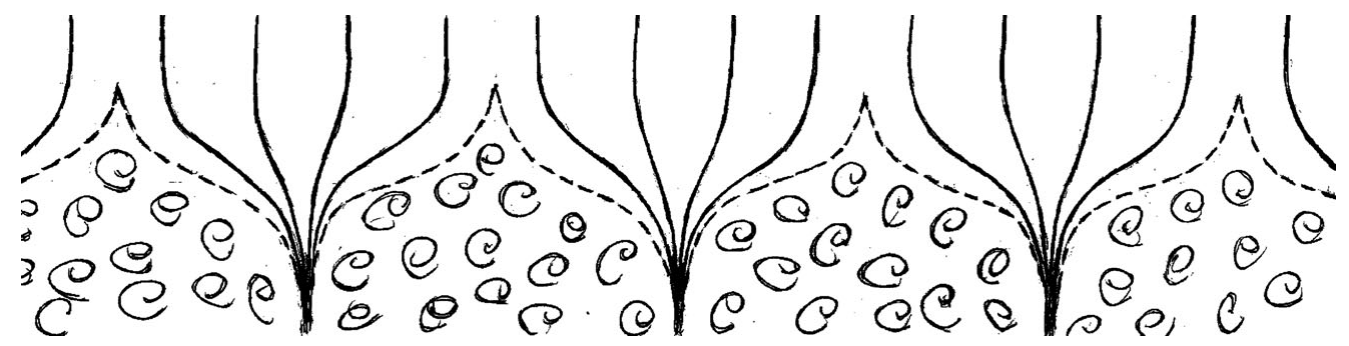

Figure 1. Standard model of quiet-sun magnetism (here illustrated for the case when the different flux tubes have the same polarity). This dualistic scenario describes the atmosphere in terms of two components, one representing the flux tubes, the other the tangled fields in between. With the Zeeman effect we see the flux tubes but not the turbulent field, with the Hanle effect the situation is the opposite. The dashed lines mark the canopy and interface between the two components.

was therefore only with the advent of highly sensitive polarimeters that this type of polarization could be fully revealed. The breakthrough came with the implementation of the ZIMPOL (Zurich Imaging Polarimeter) technology in 1994, which allowed imaging polarimetry with a precision of $10^{-5}$ in the degree of polarization (Povel 1995, 2001; Gandorfer et al. 2004). At this level of sensitivity everything is polarized, even in the absence of magnetic fields. It came as a big surprise, however, that the polarized spectrum was so richly structured, as richly as the ordinary intensity spectrum but without resembling it. It was as if a new spectral face of the Sun had been unveiled, and we had to start over to identify the various spectral structures and their physical origins. It was therefore natural to call this new and unfamiliar spectrum the "Second Solar Spectrum" (Ivanov 1991; Stenflo \& Keller 1997). A spectral atlas of the Second Solar Spectrum from 3160 to $6995 \AA$ has been published in three volumes (Gandorfer 2000, 2002, 2005).

Most of the fundamental physical processes that govern the polarized structures in the Second Solar Spectrum have by now been identified in terms of a multitude of previously unfamiliar phenomena, although we still have a long way to go for detailed quantitative modelling (cf. Stenflo 2004). Examples of identified physics are quantum-mechanical interference between atomic states of different total angular momentum quantum numbers (Stenflo 1980), hyperfine structure and isotope effects (Stenflo 1997), optical pumping that creates ground-state atomic polarization (Trujillo Bueno \& Landi Degl' Innocenti 1997; Manso Sainz \& Trujillo Bueno 2003, 2007), and molecular scattering (Stenflo \& Keller 1996, 1997; Berdyugina, Stenflo, \& Gandorfer 2002). Still there are polarization features that seem to be at odds with quantum mechanics as we know it, and which so far have eluded all attempts at a physical explanation, like the polarization peak observed at the cores of the $\mathrm{D}_{1}$ lines of sodium and barium, lines that are supposed to be intrinsically unpolarizable (Stenflo 2008).

\subsection{Signatures of the Hanle effect}

The Second Solar Spectrum exists regardless of whether there are magnetic fields around or not. The shapes and amplitudes of the various structures are however modified by magnetic fields, and it is this modification that goes under the name "Hanle effect", discovered by Wilhelm Hanle in Göttingen in 1923 (Hanle 1924; Moruzzi \& Strumia 1991). His discovery played a significant role in the early development of quantum mechanics, since it demonstrated the fundamental concept of linear superposition of quantum states. The Second Solar Spectrum is the astrophysical playground for the Hanle effect.

A scattering transition between different $J$ states consists of a coherent superposition of the scattering transitions between all the possible $m$-state combinations. The phase 
coherence between these various scattering amplitudes leads to the polarization of the scattered radiation. A magnetic field brings decoherence by shifting the frequencies of the different $m$-state transitions so that they get out of phase. The term "Hanle effect" covers all the polarization phenomena associated with the partial decoherence in a magnetic field.

A rather intuitive understanding of the Hanle effect is provided by the model of a classical oscillator in a magnetic field. Let us consider $90^{\circ}$ scattering. The incident radiation excites dipole oscillations in the transverse plane. When viewed from the $90^{\circ}$ scattering direction, the transverse plane of the incident radiation projects out to become a line. Therefore, when projected on the transverse plane of the scattered radiation, the dipole oscillation is limited to the direction perpendicular to the scattering plane, implying that the emitted radiation becomes $100 \%$ linearly polarized along that direction. If we introduce a magnetic field along the scattering direction, the dipole oscillation performs a Larmor precession around the magnetic field while being radiatively damped. The trajectory of the oscillation then forms a Rosette pattern as illustrated in Fig. 2. The spectral polarization properties of the scattered radiation are obtained by transforming the Rosette patterns to the Fourier domain, which is the domain used by quantum mechanics with its energy levels.

The form of the Rosette pattern depends on the relative magnitudes of the precession and damping rates. For weak magnetic fields the precession is slow, so the oscillation decays before much precession has happened, and the scattering polarization does not deviate much from the non-magnetic case. For strong magnetic fields the precession has time to randomize the pattern and make it more isotropic before the oscillator has decayed. The result is depolarization, reduction of the amount of scattering polarization. In the intermediate case when the precession and damping rates are similar in magnitude, we get partial depolarization combined with a net rotation of the plane of linear polarization. The Hanle effect manifests itself differently for other orientations of the magnetic field, but qualitatively the main two effects are depolarization and rotation of the plane of polarization.

Figure 2 also illustrates what Hanle polarization signatures really look like in the Sun's spectrum, and how they differ from the Zeeman effect signatures. Since the Hanle effect is a coherency phenomenon that only affects the aspect of the line formation process that is due to coherent scattering, the Hanle effect is prominent in lines for which coherent scattering plays a dominating role. In the illustrated example, recorded with ZIMPOL at NSO / Kitt Peak, the strong, resonant Ca I $4227 \AA$ line represents such a case, while the surrounding blend lines do not show any significant scattering polarization but instead depolarize the Ca I line wings and the continuum polarization. While the $4227 \AA$ line is subject to both the Hanle and Zeeman effects, the surrounding lines only show the magnetic signatures of the Zeeman effect.

The sensitivity range for the Hanle effect depends on the ratio between the Zeeman splitting and the damping width, while the polarization response of the Zeeman effect is related to the comparison of the Zeeman splitting with the spectral line width, which is mainly determined by the Doppler width. Since the damping width is smaller than the Doppler width by typically a factor of 30 , the Hanle effect is sensitive to much weaker fields than the Zeeman effect.

\subsection{Resolved fields and unresolved mixed-polarity fields}

Dramatic variations of the scattering polarization along the spectrograph slit in both Stokes $Q$ and $U$, as shown in Fig. 2, are only found in strong chromospheric lines. In contrast, the scattering polarization in photospheric lines like the often used Sr I $4607 \AA$ 


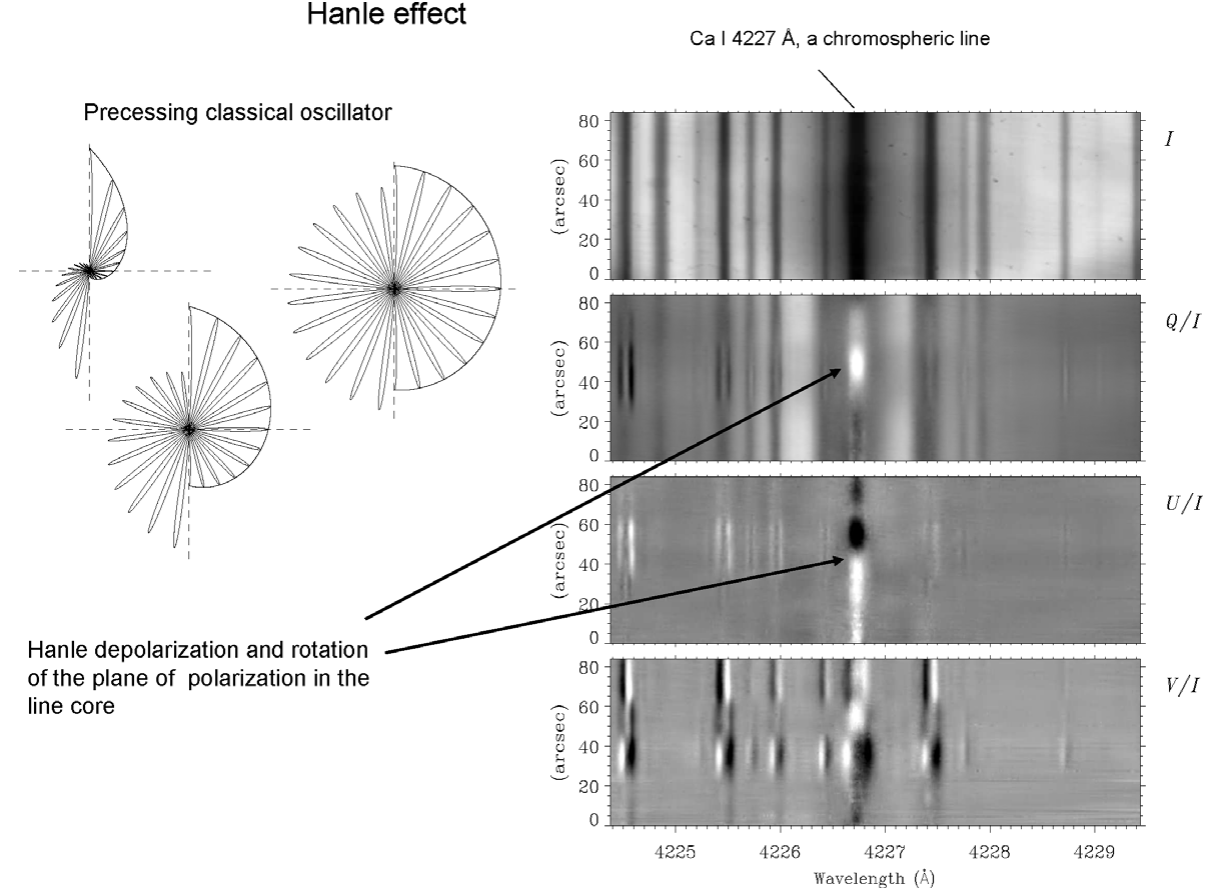

Figure 2. Left diagram: Rosette patterns formed by the trajectories of a classical dipole oscillator in a magnetic field along the line of sight, illustrating the Hanle depolarization and rotation effects. As the field strength increases, the pattern becomes more isotropic. Right diagram: Recording of the Stokes vector (the four parameters intensity $I$ and the fractional polarizations $Q / I, U / I$, and $V / I)$ with the spectrograph slit across a weakly magnetic region 20 arcsec inside and parallel to the west solar limb (at $\mu=0.20$, where $\mu$ is the cosine of the heliocentric angle). The Hanle signatures are seen in Stokes $Q$ and $U$ in the core of the Ca I $4227 \AA$ line, while the surrounding lines exhibit the characteristic signatures of the transverse Zeeman effect. In Stokes $V$ all the lines show the anti-symmetric signatures of the longitudinal Zeeman effect.

line or molecular lines like the CN lines shown in Fig. 3, exhibits little if any spatial variations in Stokes $Q$, and rarely displays significant signatures of Hanle rotation in Stokes $U$. The reason is not that the chromosphere is more magnetically structured than the photosphere. On the contrary, detailed analysis reveals that the photosphere is full of magnetic fields in the sensitivity range for the Hanle effect, but that these fields are structured mainly on spatial scales much smaller than the angular resolution of current Hanle-effect observations (a few arcsec, due to the long integration times needed to reach the required polarimetric precision).

Let us consider the hypothetical case of a small-scale turbulent magnetic field, for which the many unresolved magnetic elements within each resolution element have a random, isotropic distribution of their field vectors. While such a field may contain a large amount of magnetic energy, it does not carry much net magnetic flux after averaging over the spatial resolution elements of our instrument. It would therefore be invisible in magnetograms, due to cancellations of the plus and minus contributions to the circular polarization from the opposite polarities. Such cancellation also occurs for the Hanle rotation signatures in Stokes $U$, since the Hanle rotation effect has the same symmetry between plus and minus as the longitudinal Zeeman effect. In contrast, the Hanle depolarization effect does not suffer from such cancellations, since the depolarization only 

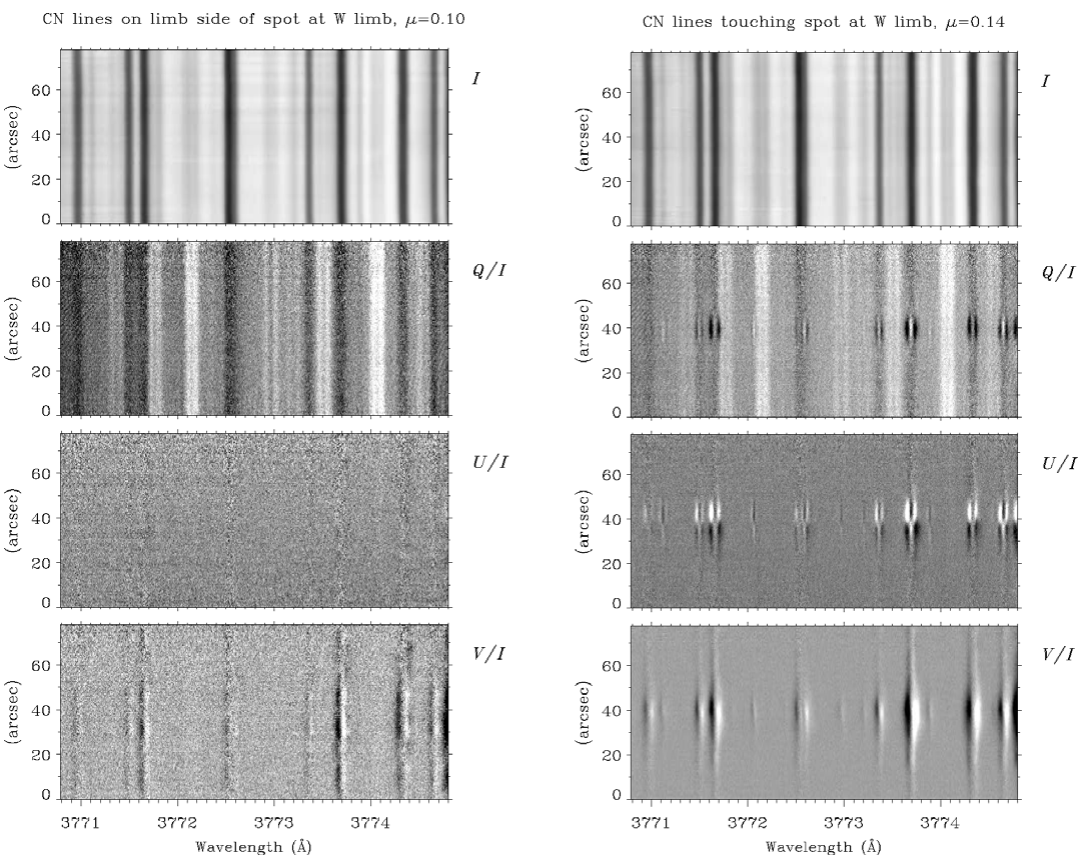

Figure 3. Example of the behavior of molecular lines in the Second Solar Spectrum. The bright, emission-like bands in Stokes $Q / I$ are due to scattering polarization by the CN molecule. Note that there is no scattering polarization in $U / I$ and no significant variation of $Q / I$ along the slit, in contrast to the surrounding atomic lines, which exhibit the familiar signatures of the transverse and longitudinal Zeeman effect. The recording was made with ZIMPOL at NSO / Kitt Peak inside the west solar limb (Stenflo 2007).

has one sign (reduction of the scattering polarization amplitude), regardless of the sign of the field orientation. The "hidden" field can therefore be revealed if we can determine the amount of Hanle depolarization that it causes.

Detailed analysis of Hanle-effect observations in photospheric lines (cf. Sect. 3 below) show that we indeed have lots of Hanle depolarization indicating the presence of large amounts of photospheric flux that is not seen in magnetograms. The circumstance that the same observations show very little sign of any Hanle rotation signals in Stokes $U$, and little variation of Stokes $Q$ along the slit, implies that the determined Hanle depolarization effect must be due to a spatially unresolved distribution of magnetic fields with magnetic elements that are much smaller than the spatial resolution used. If the tangled field were partially resolved, then the cancellation effects within each spatial resolution element would be incomplete, and we would expect to see net Hanle rotation effects and varying net depolarization effects along the slit. The observed absence of these effects is evidence for tangled field structuring on scales much smaller than a few arcsec.

\section{Diagnostics of the hidden magnetic flux}

To determine the amount of Hanle depolarization from a measured polarization amplitude we need to relate the observed polarization to the non-observed amplitude that we would have found in the absence of depolarizing magnetic fields. There are different ways to approach this problem: (1) Theoretically predict the expected non-magnetic scattering polarization amplitude by solving the polarized radiative transfer problem for 
a realistic model atmosphere. (2) Apply the differential Hanle effect (Stenflo, Keller \& Gandorfer 1998; Manso Sainz, Landi Degl'Innocenti \& Trujillo Bueno 2004; Berdyugina \& Fluri 2004), namely to record the scattering polarization simultaneously in several spectral lines with different sensitivities to the Hanle effect. (3) From the observed statistical distribution of polarization amplitudes, let the upper envelope to the distribution represent the non-magnetic values, since magnetic fields will only reduce, not enhance the polarization (Bianda et al. 1998, 1999).

Once we have managed to determine the empirical value of the Hanle depolarization with one of the above-mentioned methods, we can use this depolarization to constrain the properties of the hidden magnetic field. This step needs an assumed interpretational model. Since we only have one observable (if a single spectral line is used), the Hanle depolarization, our model is not allowed to contain more than one free parameter. With combinations of simultaneously observed lines, more free parameters may be possible (Stenflo, Keller \& Gandorfer 1998), which offers an avenue for future use of more sophisticated model constraints. With one free parameter the simplest and most natural choice has been the field strength of a randomly oriented field with an isotropic angular distribution, as we have mentioned above. It is however clear that the real turbulent field is far from being single-valued and should instead be governed by a continuous probability distribution function (PDF) that extends over a wide range of field strengths. The problem has been to characterize such an unknown PDF if we only have one free parameter at our disposal.

This problem is now being solved with guidance from empirical PDFs determined from magnetograms, representing the spatially resolved scales (Stenflo \& Holzreuter 2002, 2003) and from theoretical PDFs determined from numerical simulations of magnetoconvection, representing scales that are not yet resolved (Nordlund \& Stein 1990; Cattaneo 1999). The shapes of the empirical and theoretical PDFs are very similar to each other, although not identical. It is possible to represent the typical PDF by an analytical function that is characterized by one free scaling parameter that governs the stretching of the field-strength scale, and let this parameter be constrained by the requirement that the observed amount of Hanle depolarization should be reproduced.

The most elaborate effort with the highest degree of realism to interpret the available Hanle depolarization data has been based on 3-D radiative transfer with model atmospheres generated by numerical simulations of granular convection (Trujillo Bueno, Shchukina \& Asensio Ramos 2004). The magnetic field was constrained through fits of available data for the $\mathrm{Sr}$ I $4607 \AA$ line and $\mathrm{C}_{2}$ molecular lines. A field model assuming a single-valued volume-filling turbulent distribution gave a field strength of $60 \mathrm{G}$, substantially higher than previous estimates, which implies a significant amount of magnetic energy contained in this hidden field. However, when a much more realistic, continuous functional shape of the PDF was used instead, similar to the PDF shapes obtained from numerical simulations of magnetoconvection, the Hanle depolarization constraint leads to still stronger fields (of order $100 \mathrm{G}$ ) with correspondingly higher average energy densities, so high that the tangled field might even dominate the energy balance of the solar atmosphere (Trujillo Bueno, Shchukina \& Asensio Ramos 2004). Due to the model dependence, however, the question whether the turbulent field really dominates the energy balance remains controversial.

While the empirical values of the Hanle depolarization in the $\operatorname{Sr}$ I $4607 \AA$ line indicate such large magnetic energy densities for the hidden flux, the molecular lines indicate much smaller values. A solution to this apparent contradiction has been found through 3-D modelling (based on numerical hydrodynamic simulations) of the spatial distribution of the molecular abundance, which shows that the molecular lines are preferentially 

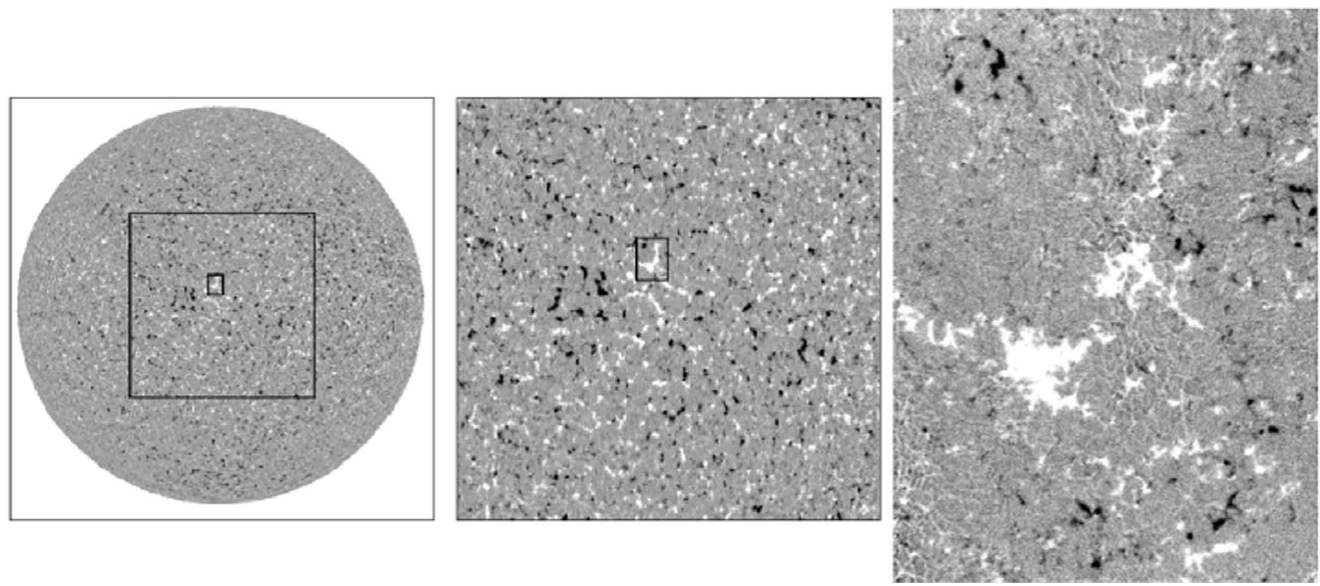

Figure 4. Zooming in on the magnetic pattern of the quiet Sun observed on 9 February 1996. The two left maps are from a Kitt Peak full-disk magnetogram, while the right, high-resolution magnetogram was recorded the same day at the Swedish La Palma Observatory (courtesy Göran Scharmer). The La Palma magnetogram covers an area that is only $0.35 \%$ of the map next to it.

formed inside the hot granules and are largely absent in the intergranular lanes (Trujillo Bueno, Shchukina \& Asensio Ramos 2004). A consistent interpretation of the observed difference in the Hanle depolarization response between the atomic and molecular lines then implies that the turbulent field is weak inside the hot granules, while the large Hanle depolarization seen in lines like Sr I $4607 \AA$ predominantly comes from the intergranular lanes. If this is indeed the case, then the turbulent field in the intergranular lanes with a relatively small filling factor must be stronger than the field derived before for a filling factor of unity. The introduction of a filling factor in the Hanle interpretation thus leads to an increase in the average magnetic energy density and strengthens the argument for an important role of the hidden magnetic flux in the overall energy balance of the Sun's atmosphere.

\section{Beyond the standard model}

The Second Solar Spectrum became accessible to observations only through the development of sufficiently sensitive imaging Stokes polarimeters, in particular with the introduction of the ZIMPOL technology, which allows the two main noise sources, seeing noise and gain table noise, to be completely eliminated. Since the only remaining noise source is the fundamental photon noise of Poisson statistics, we need larger telescope apertures to improve the $\mathrm{S} / \mathrm{N}$ ratio that we can reach with a certain temporal (integration time) and angular resolution. It is not possible to optimize spectral, spatial, temporal resolution, and polarimetric precision simultaneously, but major trade-offs between them are necessary, regardless of the size of the telescope (Stenflo 2001). The high polarimetric precision required for Hanle-effect explorations is not compatible with diffraction-limited resolution. Still the resolution of the solar granulation pattern in maps of the scatteringpolarization and the Hanle effect is within reach, (cf. Trujillo Bueno \& Shchukina 2007), but it still remains an unattained near-future challenge.

The previous dualistic magnetic-field paradigm or two-component "standard model" with a kG flux tube component with small filling factor, and a turbulent field component that is filling the remaining part of the volume, is now being replaced by a picture 
characterized by probability distribution functions (PDFs). While the strong-field tail of the distribution contains the "flux tubes" of the standard model, the bulk of the PDF corresponds to the "turbulent field" component. Instead of using two different interpretational models for the Zeeman and Hanle effects when diagnosing the spatially unresolved domain, it appears logical to apply a single, unified interpretational model based on PDFs in both cases. This has yet to be done in a consistent way.

This task is complicated by various factors. Thus the different Hanle behavior of atomic and molecular lines suggests that the PDFs are different between the inside of the granules and the intergranular lanes. To clarify this situation we need to resolve the solar granulation in Hanle effect observations. Another very important problem is that we know much less about the PDF for the angular distribution of field vectors than we know about the PDF for the vertical field strengths. Figure 4 illustrates the fractal appearance as we zoom in on the quiet-sun magnetic pattern at the center of the solar disk. The pattern looks similar on all scales, with a coexistence of weak and strong fields over a wide dynamic range. The PDF for the vertical field-strength component is nearly scale invariant and can be well represented by a Voigt function with a narrow Gaussian core and "damping wings" extending to kG values (Stenflo \& Holzreuter 2002, 2003). A fractal dimension of 1.4 has been found from both observations and numerical simulations (Janssen, Vögler \& Kneer 2003). The simulations indicate that this fractal behavior extends well into the spatially unresolved domain.

The richly structured Second Solar Spectrum with its many novel magnetic-field effects opens a new window to explorations of previously inaccessible aspects of the Sun. With the vast amounts of hidden magnetic energy in the spatially unresolved magnetoconvective spectrum, the determination of the properties of the hidden field has become a central task for contemporary solar physics.

\section{References}

Berdyugina, S. V. \& Fluri, D. M. 2004, $A \& A 4417,775$

Berdyugina, S. V., Stenflo, J. O., \& Gandorfer, A. 2002, A\& $A$ 388, 1062

Bianda, M., Solanki, S. K., \& Stenflo, J. O. 1998, A\& $A$ 331, 760

Bianda, M., Stenflo, J. O., \& Solanki, S. K. 1999, A\&A 350, 1060

Cattaneo, F. 1999, ApJ 525, L39

Gandorfer, A. 2000, The Second Solar Spectrum, Vol. I: $4625 \AA$ to $6995 \AA$, ISBN no. 372812764 7 (Zurich: VdF)

Gandorfer, A. 2002, The Second Solar Spectrum, Vol. II: $3910 \AA$ to $4630 \AA$, ISBN no. 37281 28554 (Zurich: VdF)

Gandorfer, A. 2005, The Second Solar Spectrum, Vol. III: $3160 \AA$ to $3915 \AA$, ISBN no. 37281 30184 (Zurich: VdF)

Gandorfer, A. M., Povel, H. P., Steiner, P., Aebersold, F., Egger, U., Feller, A., Gisler, D., Hagenbuch, S., \& Stenflo, J. O. 2004, A\&A 422, 703

Hanle, W. 1924, Z. Phys. 30, 93

Ivanov, V. V. 1991, in: L. Crivellari, I. Hubeny, \& D. G. Hummer (eds.), Stellar Atmospheres: Beyond Classical Models (Dordrecht: Kluwer), Proc. NATO, pp. 81

Janssen, K., Vögler, A., Kneer, F. 2003, A\&\&A 409, 1127

Manso Sainz, R. \& Trujillo Bueno, J. 2003, Phys. Rev. Letters 91, 111102

Manso Sainz, R. \& Trujillo Bueno, J. 2007, in: P. Heinzel, I. Dorotovic, \& R. J. Rutten (eds.), The Physics of Chromospheric Plasmas, ASP Conf. Ser. (San Francisco: ASP), vol. 368, 155

Manso Sainz, R., Landi Degl'Innocenti, E., \& Trujillo Bueno, J. 2004, ApJ 614, L89

Moruzzi, G. \& Strumia, F. (eds.) 1991, The Hanle Effect and Level-Crossing Spectroscopy (New York: Plenum) 
Nordlund, Å. \& Stein, R. F. 1990, in: J. O. Stenflo (ed.), Solar Photosphere: Structure, Convection, and Magnetic Fields, IAU Symp. 138, 191-211

Povel, H. 1995, Optical Engineering 34, 1870

Povel, H. 2001, in: G. Mathys, S. K. Solanki \& D. T. Wickramasinghe (eds.), Magnetic Fields Across the Hertzsprung-Russel Diagram, ASP Conf. Ser. (San Francisco: ASP), vol. 248, pp. $543-552$

Rüedi, I., Solanki, S. K., Livingston, W., Stenflo, J. O. 1992, A $\& A$ 263, 323

Solanki, S. K. 1993, Space Sci. Rev. 63, 1

Spruit, H. 1976, SP 50, 269

Stenflo, J. O. 1973, SP 32, 41

Stenflo, J. O. $1980, A \xi \mathcal{G} A 4,68$

Stenflo, J. O. 1982, SP 80, 209

Stenflo, J. O. 1997, A\&A 324, 344

Stenflo, J. O. 2001, in: G. Mathys, S. K. Solanki \& D. T. Wickramasinghe (eds.), Magnetic Fields Across the Hertzsprung-Russel Diagram, ASP Conf. Ser. (San Francisco: ASP), vol. 248, pp. 639

Stenflo, J. O. 2004, Rev. Mod. Astron. 17, 269

Stenflo, J. O. 2007, Memorie della Societa Astronomica Italiana 78, 181

Stenflo, J. O. 2008, in: S. Berdyugina, K. N. Nagendra, \& R. Ramelli (eds.), Solar Polarization, Proc. 4th SPW, ASP Conf. Ser. (San Francisco: ASP), in press

Stenflo, J. O. \& Holzreuter, R. 2002, in: H. Sawaya-Lacoste (ed.), Magnetic Coupling of the Solar Atmosphere, ESA Publ. SP-505, 101

Stenflo, J. O. \& Holzreuter, R. 2003, in: A. A. Pevtsov \& H. Uitenbroek (eds.), Current Theoretical Models and High Resolution Solar Observations, Proc. 21st International NSO/SP Workshop, ASP Conf. Ser. (San Francisco: ASP), vol. 286, 169

Stenflo, J. O. \& Keller, C. U. 1996, Nature 382, 588

Stenflo, J. O. \& Keller, C. U. 1997, A\&BA 321, 927

Stenflo, J. O. \& Lindegren, L. 1977, A\&A 59, 367

Stenflo, J. O., Harvey, J. W., Brault, J. W., \& Solanki, S. K. 1984, A\&GA 131, 333

Stenflo, J. O., Keller, C. U., \& Gandorfer, A. 1998, A\& A 329, 319

Trujillo Bueno, J. \& Landi Degl' Innocenti, E. 1997, ApJ 482, L183

Trujillo Bueno, J. \& Shchukina, N. 2007, ApJ 664, L135

Trujillo Bueno, J., Shchukina, N., \& Asensio Ramos, A. 2004, Nature 430, 326

\section{Discussion}

STRASSMEIER: If you were to observe the "Sun as a Star", wouldn't the Stokes-Q signal due to the coherent scattering cancel out? And, if you would ignore it, would it cross talk into Stokes V?

Stenflo: For stars with axially symmetric stellar disks the effect would indeed cancel out. However, for objects with significant deviations from axial symmetry there may be net observable scattering polarization signatures. Apart from cross talk produced by the instrument used, there should not be any other kind of Q vs. V cross talk that one needs to be concerned about.

Kouтснму: Is it possible to observe the Second Solar Spectrum using FTS instruments? If not, why? If yes, why it is not done?

Stenflo: My first encounter with the Second Solar Spectrum was in 1978-79 when I made a survey at the scattering polarization from the deep UV (3160 ̊) to the near infrared. For the wavelengths above $4200 \AA$ I used the FTS at Kitt Peak. However, as the noise level was about $0.1 \%$ in the degree of linear polarization, I only saw the "tips of the icebergs" at the Second Solar Spectrum, the majority of the structures were down in 
the noise. It did not seem possible to push the noise much below $0.1 \%$ with the FTS when it was used, as we did, with $1000-\AA$ wide prefilters. Limiting the simultaneous spectral coverage would help the noise, but this avenue was never pursued (which is unfortunate).

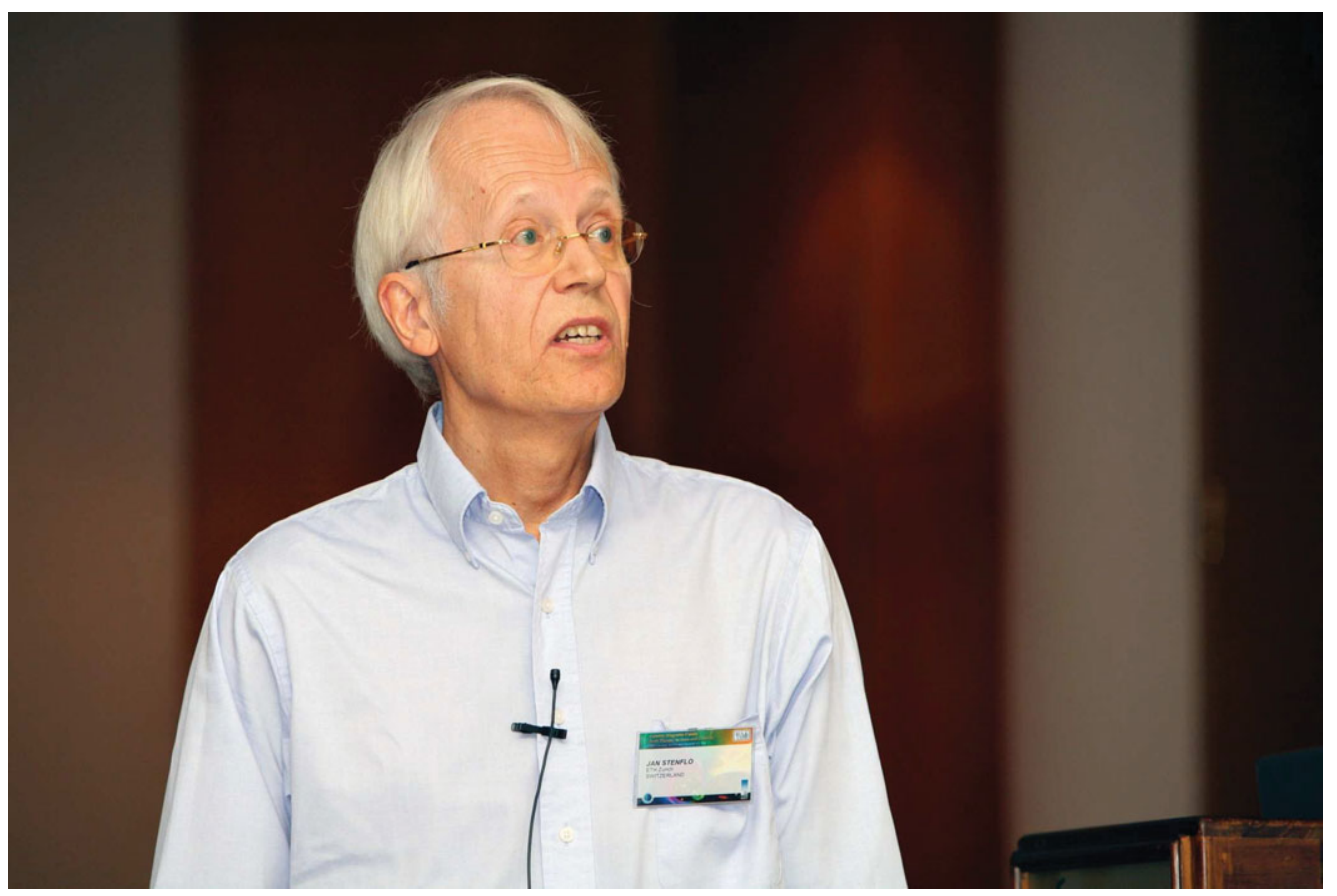

Jan Stenflo and ...

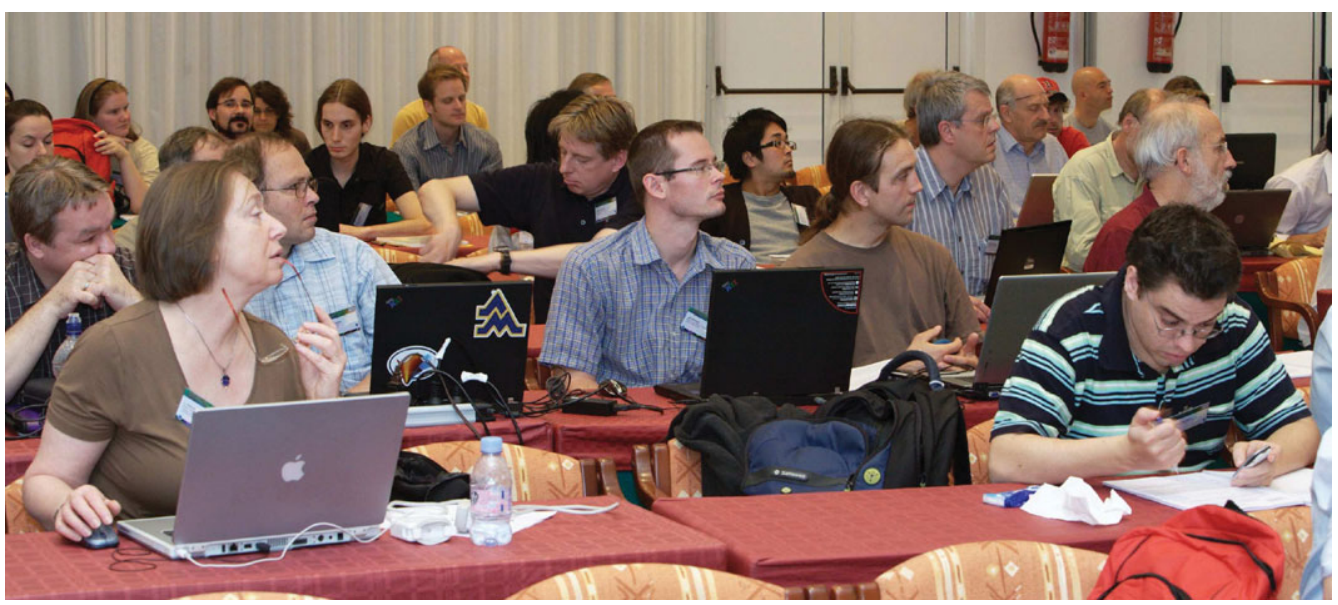

... a very interested auditorium 


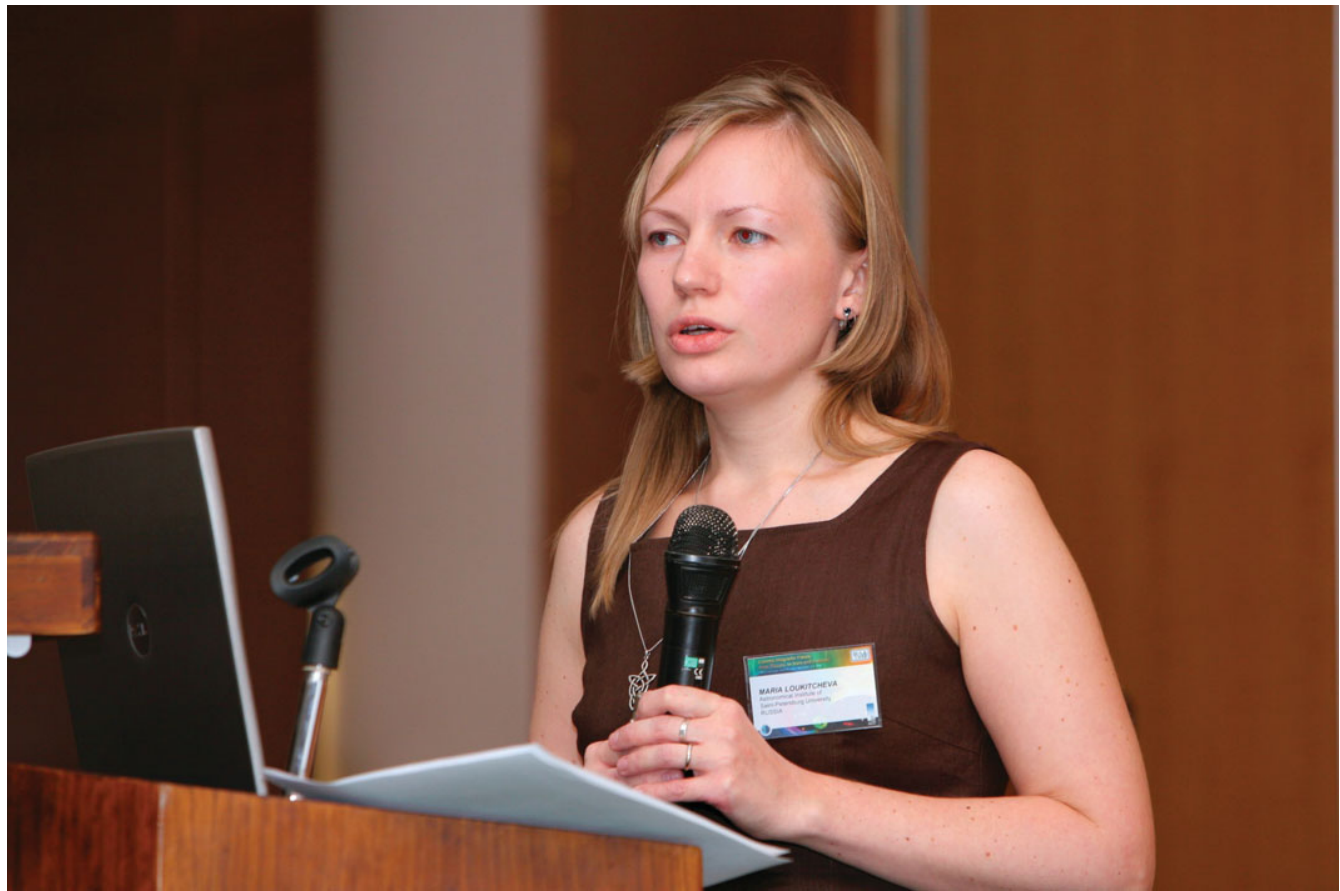

Maria Loukitcheva

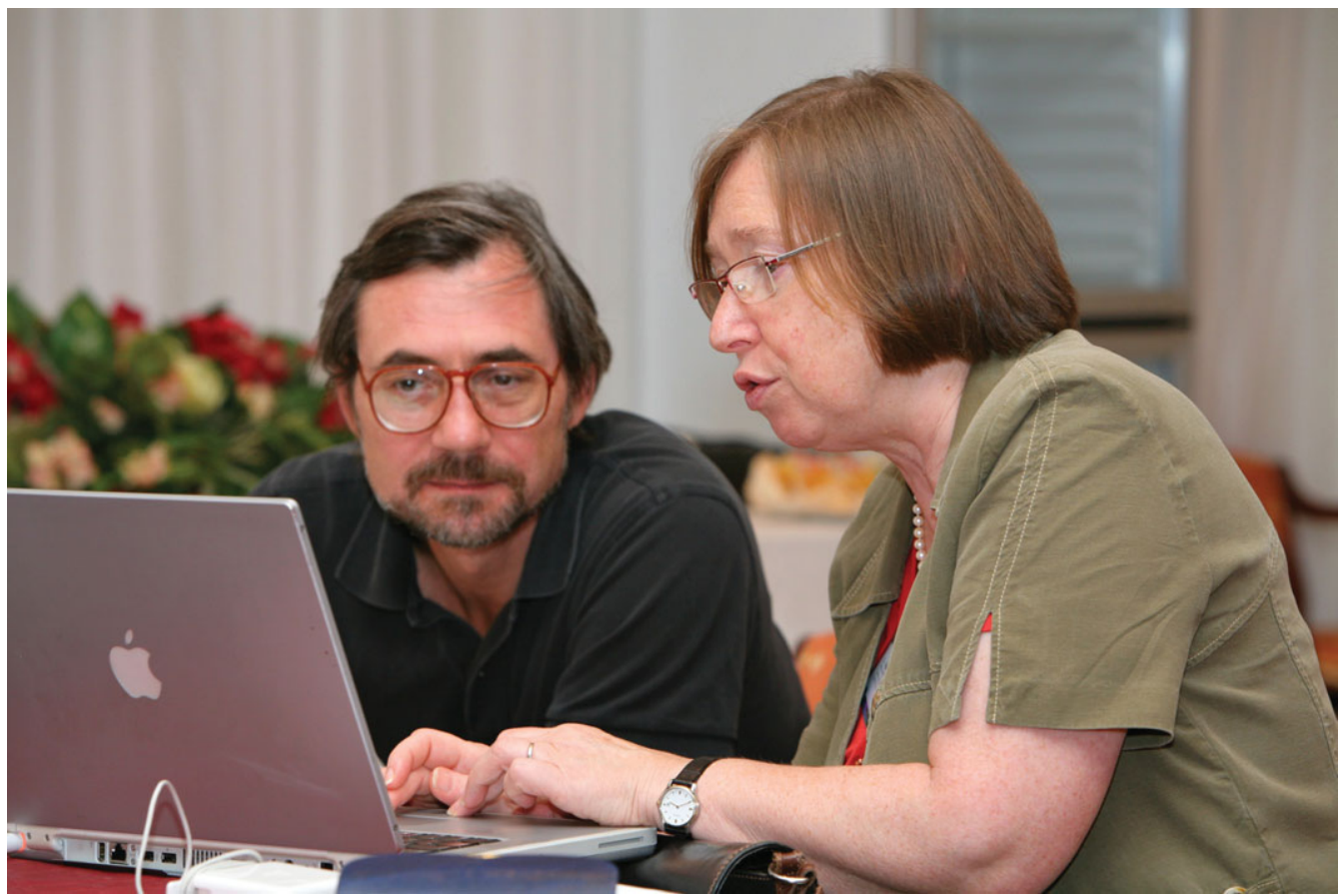

Ilya llyin (left) and Lidia van Driel-Gesztelyi 